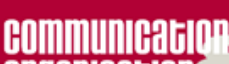

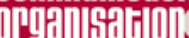

Communication et organisation

Revue scientifique francophone en Communication

organisationnelle

$54 \mid 2018$

Pratiques de la communication et Big Data

\title{
Ryan S. BISEL, Organizational Moral Learning: a Communication Approach
}

New York, NY : Routledge, 2017

\section{Delphine Dupré}

\section{(2) OpenEdition}

Journals

Édition électronique

URL : https://journals.openedition.org/communicationorganisation/7357

DOI : 10.4000/communicationorganisation.7357

ISBN : 979-10-300-0341-3

ISSN : $1775-3546$

Éditeur

Presses universitaires de Bordeaux

\section{Édition imprimée}

Date de publication : 1 décembre 2018

Pagination : 262-264

ISBN : 979-10-300-0340-6

ISSN : 1168-5549

\section{Référence électronique}

Delphine Dupré, «Ryan S. BISEL, Organizational Moral Learning: a Communication Approach »,

Communication et organisation [En ligne], 54 | 2018, mis en ligne le 01 décembre 2018, consulté le 04 janvier 2023. URL : http://journals.openedition.org/communicationorganisation/7357 ; DOI : https:// doi.org/10.4000/communicationorganisation.7357 


\section{Ryan S. BISEL, Organizational Moral Learning: $A$ C Communication Approach. - New York, NY : Routledge, 2017. ISBN 978-1-138- 11956-7, $40 €$}

Chercheur en communication, Ryan S. Bisel étudie les phénomènes organisationnels relatifs à l'éthique. Le point de départ de sa réflexion réside dans deux constats : tout d'abord, une revue de la littérature scientifique l'amène à considérer que les formations à l'éthique - dispensées tant dans le cadre universitaire que dans les organisations sont inefficaces, au sens où elles ne contribuent que faiblement à favoriser des comportements conformes à l'éthique.

La même conclusion s'impose concernant les chartes éthiques. Ces dernières sont souvent édictées a posteriori, afin de reconstruire la réputation et d'apporter un cadrage aux comportements. De plus, rassurés par l'existence de ces chartes, les managers tendraient à délaisser leurs actions de sensibilisation et de prévention quotidiennes.

Pour comprendre l'échec de ces chartes ainsi que des formations à l'éthique, l'auteur recourt aux avancées récentes en neurosciences. Des expérimentations menées dernièrement suggèrent que les êtres humains disposent, dès le plus jeune âge, d'intuitions morales spontanées. Toutefois, que nos cerveaux soient programmés pour distinguer le bien du mal s'avère insuffisant pour garantir des raisonnements, des décisions et des actions éthiques. Selon l'auteur, les phénomènes relatifs à l'éthique sont principalement culturels.

Dans les organisations, nos intuitions morales «spontanées " sont supplantées par l'exigence de conformité à un ensemble de normes relationnelles, comportementales et communicationnelles. La force de ces dernières procèdent de leur invisibilité aux yeux des individus sur lesquels elles agissent. De plus, le système de normes organisationnel peut facilement s'effacer derrière des rationalisations managériales, telles que la recherche d'efficacité, la quête du profit, la satisfaction des parties prenantes, etc.

L'idée essentielle défendue par l'auteur est que c'est par la communication que les normes qui structurent les conduites éthiques émergent, se renforcent et s'institutionnalisent. L'auteur étaye sa démonstration en s'inspirant de la pensée de Karl Weick et du courant de la communication constitutive des organisations (CCO). Il mobilise le concept "d'effet motus " pour évoquer les contraintes qui pèsent sur les acteurs et les empêchent de parler d'éthique au quotidien. En effet, dénoncer 
les pratiques contraires à l'éthique est considéré comme une transgression des normes de politesse, car perçu comme moralisateur. De plus, ces discussions délicates menacent de mettre en péril tant la face des interlocuteurs que la relation qu'ils entretiennent.

L'auteur précise que le statut des interactants n'est pas neutre. La réticence à parler d'éthique est accrue lorsque l'interlocuteur est un manager. Tout salarié faisant remonter une mauvaise nouvelle à son supérieur hiérarchique risque d'être identifié à cette dernière. Désapprouver une décision ou une pratique en invoquant des raisons morales peut être perçu comme une forme de déloyauté, susceptible d'entrainer des sanctions. Enfin, ce silence s'expliquerait par l'influence potentiellement néfaste que le manager peut exercer sur la carrière de ses subordonnés.

Les différentes enquêtes menées par l'auteur lui ont permis d'observer les tactiques de communication mises en ouvre par les subordonnés afin d'évoquer avec leurs managers des sujets relatifs à l'éthique sans se mettre en danger. Souvent, ces derniers tendent à enrober leurs propos en recourant à des justifications managériales ou juridiques. L'auteur constate également l'usage fréquent des euphémismes. Il note que plus un sujet est délicat, plus il est exprimé de manière "feutrée ». Certes, ce « silence moral » préserve la relation, mais il alimente un cercle vicieux de tolérance à l'égard des pratiques et décisions potentiellement condamnables.

Dans la dernière partie de l'ouvrage, l'auteur propose des pistes de réflexion en vue de façonner un " apprentissage moral ", c'est-à-dire d'améliorer la capacité des organisations à identifier les manquements éthiques et à s'adapter pour les prévenir. Par exemple, les formations à l'éthique sont peu efficaces car, soit elles se résument à des raisonnements philosophiques abstraits, soit elles sont déconnectées des réalités du terrain. Or, dans leur travail, les individus ne sont pas confrontés à des choix binaires entre le bien et le mal. Au contraire, ils doivent composer avec de véritables dilemmes éthiques. L'auteur préconise de préserver du temps et d'instaurer des espaces de dialogue pour permettre aux agents de s'adonner à des discussions incarnées sur ces dilemmes. Il conseille également d'accroître les compétences communicationnelles des managers. Ces derniers devraient se montrer attentifs aux feedbacks de leurs équipes, et ne pas récompenser systématiquement ceux qui se conforment à la règle du silence. 
Pour conclure, la visée normative de l'ouvrage n'empêche pas l'auteur de proposer une analyse critique des mécanismes communicationnels et culturels qui instaurent un véritable « silence moral ». L'intérêt de l'ouvrage réside dans son ancrage pluridisciplinaire, l'auteur parvenant à articuler des apports issus de la psychologie sociale, des neurosciences et des Sciences de l'Information et de la Communication.

Delphine DUPRÉ

Delphine Dupré est doctorante contractuelle au sein du laboratoire MICA (EA 4426) de l'Université Bordeaux Montaigne. Sa thèse porte sur les incivilités par messagerie électronique et s'inscrit dans le cadre du projet régional Civilinum coordonné par Valérie Carayol et Aurélie Laborde.

\section{Suzanne MCCORKLE et Melanie REESE, Personal conflict management: theory and practice. New York : Routledge, 2018. ISBN 978-1-138-21099-8, 57 €.}

Cet ouvrage, dont les auteures sont à la fois chercheuses et médiatrices, constitue une mise en perspective pédagogique de leurs expériences professionnelles ainsi que des avancées scientifiques en gestion des conflits.

La première partie consiste en un cadrage du concept de « conflit interpersonnel ». Selon les auteures, un conflit intervient lorsqu'au moins deux personnes font l'expérience de difficultés relationnelles dont l'élément déclencheur est la présence de buts contradictoires. Elles en distinguent 4 catégories : les buts qui menacent la face des interactants (face goals), ceux qui émergent de points de vue divergents sur la manière d'accomplir une tâche (process goals), les buts relatifs à la répartition des ressources (substantive goals) et ceux portants sur des aspects relationnels (relationship goals).

Les auteurs identifient deux principales approches relatives à la gestion des conflits : celle de la compétition et celle de la coopération. Alors que, dans l'approche compétitive, le principal enjeu réside dans la position des interactants, c'est-à-dire le fait de sortir vainqueur ou non du conflit, les méthodes plus coopératives sont orientées vers la recherche d'un terrain d'entente et visent à satisfaire les intérêts des deux parties. 